\title{
Enhancing Real Time Heavy Metal Detection using Embedded Portable Signal Processing Unit
}

\author{
${ }^{1}$ Amin Mazaheri, ${ }^{1}$ Maryam Mohd Isa, ${ }^{1}$ Roslina Mohd Sidek and ${ }^{2}$ Nor Azah Yusof \\ ${ }^{1}$ Department of Electrical and Electronic Engineering, Faculty of Engineering, \\ ${ }^{2}$ Department of Chemistry, Faculty of Science, University Putra Malaysia (UPM), \\ 43400 Serdang, Malaysia, mazaheri_amin@yahoo.com, +86-24-23986353,+86-24-23986355
}

\begin{abstract}
This study presented a low-cost embedded system designed for enhancing Anodic Striping Differential Pulse Voltammetry (ASDPV) signals and detecting heavy metals by implementation of Digital Signal Processing (DSP) unit. For the in-situ analyzing of electrochemical data obtained by ASDPV from a three-electrode cell. The system implements floating point FIR filter for de-noising of sampled signals for enhancing voltammogram peaks location. On-line multiple peak detection and Gaussian distribution algorithms have been developed onto processor for the detecting and analyzing heavy metals of various applications. Potential scanning range of this device is between -1500 and $+1500 \mathrm{mV}$ within frequency range $0.1-1 \mathrm{kHz}$ which make the device able to use for measuring wide rang concentrations of samples and environmental monitoring without the need of Personal Computer (PC) or expert user. The developed algorithms has been tested for measuring heavy metal samples with different concentration.
\end{abstract}

Key words: Heavy metal detection, electrochemical instrument, Anodic Striping Differential Pulse Voltammetry (ASDPV), Digital Signal Processing (DSP), measuring, heavy

\section{INTRODUCTION}

Today's heavy metal contaminations has a critical worry in human health, ecosystem and also economics. Many heavy metals are dangerous at even low concentration (Francis et al., 2003). They can also be harmful by accumulating in human organs lead to long-term bad health side effects such as heart disease, high blood pressure and cancer. Consequently, heavy metal monitoring and detection of trace concentration is very critical. Electrochemical systems have been extensively utilized in industrial (Kazi et al., 2016), biotechnology (Ghoreishizadeh et al., 2012), Physics and Chemistry laboratories (Honeychurch, 2012) for trace metal monitoring. The basic goals of experiment that uses these instruments are to detect, identify, monitor and analyze critical parameters of chemical reactions (Strasunske, 2010). Implemented electrochemical detection system consists of electrodes as transducer, potentiostat as electrical signal measurement and a processor for data processing block. Electrochemical sensor include three electrodes that support the chemical reactions inside a chemical cell (Wang, 2004). A potentiostat is an electronic instrument which has two main functions as shown in Fig. 1. First, controls the potential difference between the
Working Electrode (WE) and the Reference Electrode (RE) at a desired cell potential which is important for the suitable operation of the electrochemical sensor. Second, measure the current generated from the chemical reaction at the sensing electrode which is passed to the signal processing circuit for further operation and information extraction (Wang, 2004).

Several analytic, biological, biochemical and bioanalytical equipment have been designed for determination of particular or mixture compounds (Singh et al., 2008; Dorokhin et al., 2014; Cheng et al., 2002) but the majority of them suffer from the impossibility to miniaturize these devices. Analysis in environment needs one other requirement which is measuring of a large number of samples in short time. Due to the above mentioned points developing and suggesting of simple analytical instruments, methods and procedures with low detection limits and offering online and in-situ monitoring of environment are needed.

Traditionally, a man will visually detect tops and bottoms of peaks on a graph, manually measure the height and assume that the relationship between peak height and concentration is linear. With the advancement of microprocessors in-situ electrochemical system provides feasible solution for sensitive detection and miniaturized

Corresponding Author: Amin Mazaheri, Department of Electrical and Electronic Engineering, Faculty of Engineering, University Putra Malaysia (UPM), 43400 Serdang, Malaysia, mazaheri_amin@yahoo.com, +86-24-23986353, +86-24-23986355 


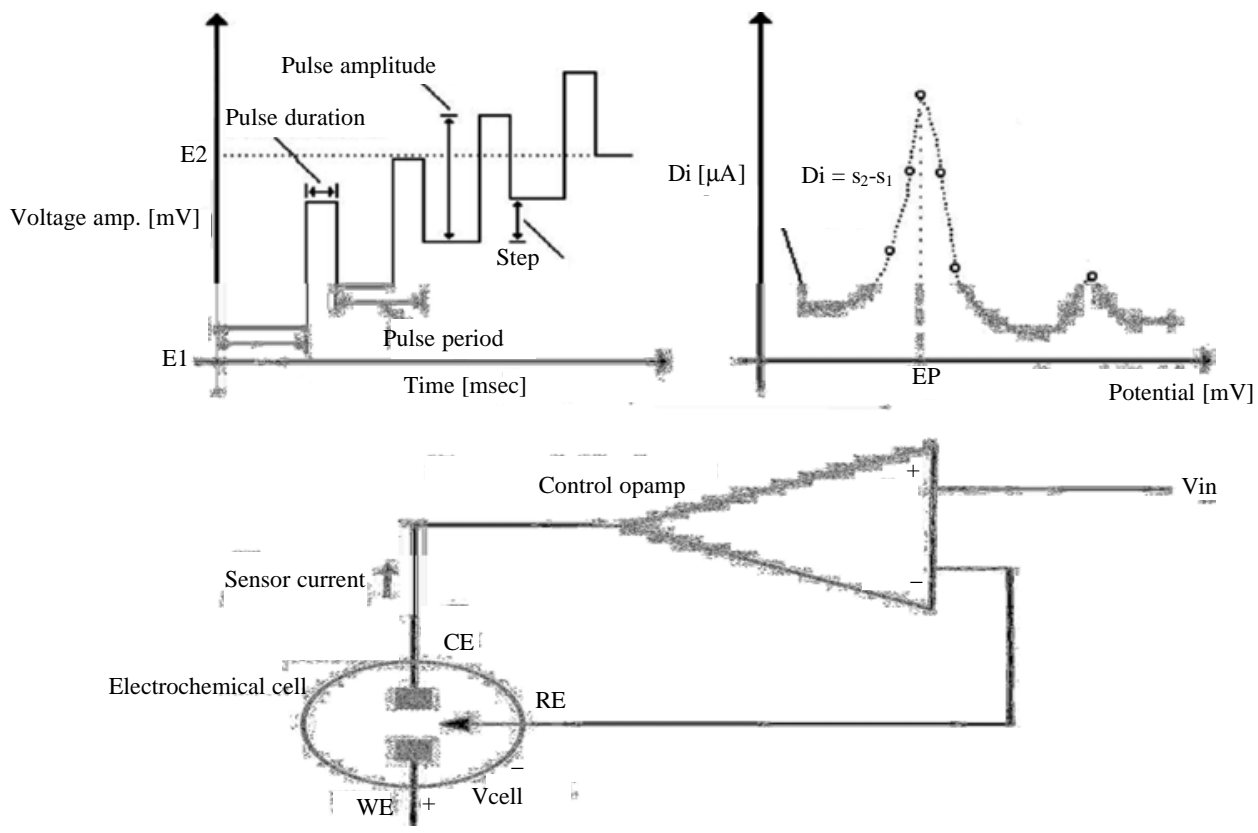

Fig. 1: a) Anodic striping differential pulse voltammetry excitation signal and its resulting voltammogram and b) A basic potentiostat

platform. The instruments currently used for the analysis of samples are highly-priced units and required trained personnel to carry out the analysis and to understand the data. These analytical systems are also too bulky to be used in-situ. In many cases, the longtime delays involved in this process are unacceptable and online processing is required.

In this study, the development of a Digital Signal Processing (DSP) unit for online processing of ASDPV-based data is described. The DSP calculations are embedded in hardware using an ARM Cortex-m3 processor from NXP Company (LPC1 768) and interfaced with an LCD that can communicate with PC through UART port. For scanning the solution, a differential pulse waveform generator is developed. The functionality of the DSP unit and other part of the system is successfully evaluated with the chemical experiments.

\section{MATERIALS AND METHODS}

Fundamental of Electrochemistry and ASDPV: Electro-analytical techniques are concerned with the interplay between electricity and Chemistry, namely the measurement of the electrical quantities such as potential, current and their relationship to chemical parameters (Zoski, 2007). Using of electrical measurements for analytical purpose has found a vast range of application including industrial control, environmental monitoring and biomedical analysis. Under equilibrium and in the absence of an externally applied voltage, a single polarizable electrode resting in solution and develop a potential based on the ratio of the solution's chemical species. This potential is given by the Nernst equation (Bard and Faulkner, 1980):

$$
\mathrm{E}=\mathrm{E}^{0}-\frac{\mathrm{RT}}{\mathrm{nF}} \ln \left(\frac{\mathrm{C}_{\mathrm{O}}}{\mathrm{C}_{\mathrm{R}}}\right)
$$

Where:

$\mathrm{E}^{0}=$ The standard potential for redox reaction (oxidation and reduction reaction)

$\mathrm{R}=$ The molar gas constant $\left(8.314 \mathrm{JK}^{-1} \mathrm{~mol}^{-1}\right)$

$\mathrm{n}=$ The number of electrons transferred in reaction

$\mathrm{T}=$ The Kelvin Temperature

$\mathrm{F}=$ The Faraday's constant (96.487 coulombs)

$\mathrm{C}_{0}=$ The Concentration of the oxidized form of the species

$C_{R}=$ The Concentration of the reduced form of the species

When a voltage sufficiently larger than this equilibrium potential (an over-potential) is applied to the electrode, this forces the system out of equilibrium and results in a reduction/oxidation (redox) reaction of the following form (Bard and Faulkner, 1980). Redox (oxidation and reduction) reaction is defined as reaction in which electrons are transferred. Oxidation refers to the loss of electrons while reduction refers to the gain of electrons as in Eq. 2:

$$
\mathrm{O}+\mathrm{ne}^{-} \mathrm{R}
$$


Where:

$\mathrm{O}=$ The Oxidized form of the species

$\mathrm{n}=$ The number of electrons per molecule oxidized or reduced

$\mathrm{e}=$ An electron

$\mathrm{R}=$ The reduced form of the species

For every oxidation or reduction, $\mathrm{n}$ electrons are transferred from solution to electrode or vice versa which produce the current. The resulting current form a change in oxidation state of electroactive species is named Faradic current (IF) because it is governed by Faraday's law (the amount of chemical reaction caused by the flow of the current is proportional to the amount of electricity passed). IF is a function of the concentration of the oxidized species and the electrode area. Thus, the measured faradaic current corresponds to a specific ion concentration.

Voltammetry is one of a few systematic electrochemical technique with adequate sensitivity in the low concentration for in-situ monitoring of trace metals (Fischer et al., 2003). Voltammetry is based on an applied time-dependent potential on the electrode sensor and measuring Faradic current, of chemical analytic (Zoski, 2007). The resulting plot of measured current vs. applied potential named as voltammogram. The peaks of Faradic current response on the voltammogram is linearly proportional to chemical concentration.

Anodic Stripping Differential Pulse Voltammetry (ASDPV) is a method of voltammetry with small influence of capacitive current and improves the Faradic current by attenuating the background currents (Harvey, 2000). In this method, the excitation signal is a linear ramped, fixed value pulses that current has sampled at the end of each pulse period. The difference of this two current is plotted compared to the applied potential. The resulting voltammogram, consists of current peaks which the value of peaks are proportional to the concentration of the chemical element (Fig. 1a) (Bard and Faulkner, 1980).

However, early voltammetric techniques used only two electrodes, the modern methods makes use of a three-electrode sensors. This electrodes are named as Working Electrode (WE), Reference Electrode (RE) and Counter Electrode CE (auxiliary electrodes). The voltammetry pulses which called as excitation signal are applied over the WE and RE. The WE potential can be controlled compared to the fixed potential of the RE and measure the resulting current through the $\mathrm{WE}$ and $\mathrm{CE}$. Basically, to can apply a stable excitation signal between the reference electrode and the working electrode need an electronic device known as potentiostat. Furthermore, potentiostat measuring the generated current passing through the working electrode and a counter electrode and convert it to analog voltage. Figure $1 \mathrm{~b}$, shown the basic construction of a three-electrode sensor with a potentiostat device.

A block diagram of the proposed system architecture is demonstrated in Fig. 2. This system is consisting of a MCU with DSP ability which communicate with a potentiostat and electrochemical cell. The MCU is a 32-bit ARM Cortex-M3 based which is for embedded applications has a high level of integration and low power dissipation. It used as a miniaturized computer to identify and predict the heavy metals concentration with the performing of defined behaviors: generate excitation



Fig. 2: Block diagram of system architecture 




Fig. 3: Block diagram of system flow chart

signal based on ASDPV for potentiostat, sample the chemical measured current and processing of ASDPV voltammogram data.

The differential pulse is a waveform that consists of a symmetric square wave pulse with constant amplitude superimposed on a sweep wave having a pre-defined scanning period. Generally, the ASDPV excitation signals are the frequency range of $0.1-1 \mathrm{kHz}$ for different scan rate. In order to generate the DPV waveform, an embedded 10 bit Digital-to-Analog Converter (DAC) is used.

When the potentiostat scanning the excitation signal between $\mathrm{RE}$ and $\mathrm{WE}$, electrochemical reactions produce a small current through the sensor $\mathrm{WE}$ and $\mathrm{CE}$. While the scan voltage reaches the reduction potential of a given species, the oxidization rate increased sharply, that raised the sensor current (Faradic current) and causes a peak in the electrode current. Potentiostat measured this induced current and after conversion to the voltage, it is sampled through the embedded 12 bit Analog-to-Digital Converters (ADCs) and stored in the MCU memory.

Software and algorithms implementation: The firmware and algorithms of system for real time processing are all implemented using the native $C$ language. Figure 3 shown the block diagram of system flow chart. The states of firmware is based on Wait for Interrupt (WFI). To reduce device's current consumption and conserve power, the system remain at idle state that put the processor at the sleep mode and wait to an interrupt happen for execution
Table 1: Configuration of generated differential pulse

\begin{tabular}{ll}
\hline Variables & Values \\
\hline Deposition time & $60 \mathrm{sec}$ \\
Pulse amplitude & $30 \mathrm{mV}$ \\
Step & $3 \mathrm{mV}$ \\
Pulse period & $300 \mathrm{mV}$ \\
Pulse width & $100 \mathrm{msec}$ \\
Start potential & $-1500 \mathrm{mV}$ \\
End potential & $+1500 \mathrm{mV}$ \\
\hline
\end{tabular}

the tasks. Sleep mode halts the clock to the CPU's core, stops executing instructions and powers down the on-chip flash but its DMA, SRAM, GPIOs and Timers still remain active.

To perform the ASDPV experiments need to scan electrodes from initial to final voltage. ASDPV signal parameters are shown at Table 1. The signal generator execute instruction in a loop. It start by sending start voltage data burst from DMA to DAC during the time (pulse period-pulse width) then to complete the pulse period, data of the DAC increased by pulse amplitude and kept it at this voltage level during the pulse width time. Next pulse started by voltage level of (pulse amplitude-step). The loop stopped when voltage level reach to the END voltage value.

Data sampling and FIR filter implementation: In data acquisition with a device, generally, the noise has higher frequencies and lower amplitudes than the desired signal. At ADC sampling this noise has effect on accuracy of system. Theoretically, ASDPV need two sample at the end of each pulse of a period which single sampling is 


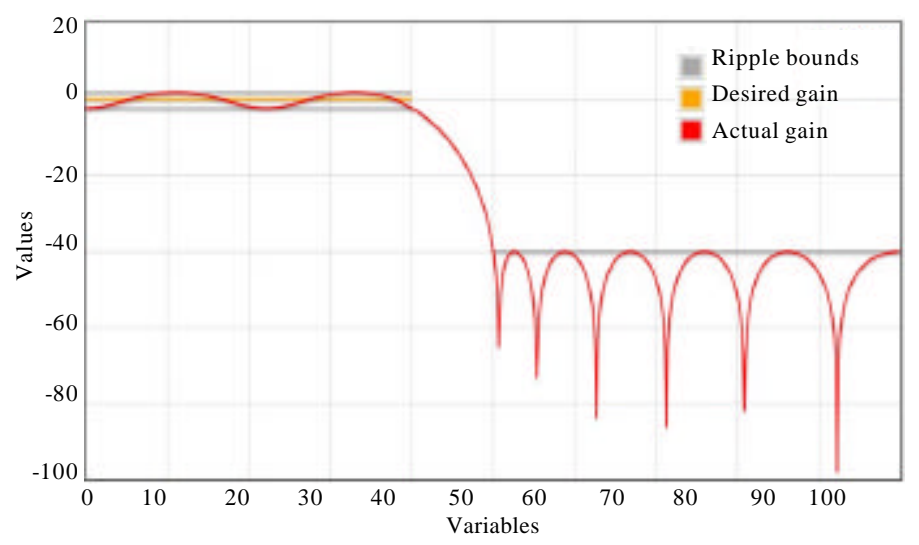

Fig. 4: The normalized frequency response of the designed FIR low-pass filter

vulnerable of noise affection. To improve the sampling accuracy, oversampling, FIR filter and then averaging the sensor signal has been implemented. At this strategy for each half cycle of pulse, the ADC takes 1024 (45) successive sampling. Every power of 4 extra sampling adds 1 bit of resolution to the ADC measurement and improves the SNR by a factor of $6 \mathrm{db}$ []. Therefore, by using this sampling technique can significantly increases the SNR and improve the ADC resolution from 12-17 bits without any additional components.

Inner-product operations are used extensively in embedded Digital Signal Processing (DSP) systems. One applications in signal processing is filtering. In embedded systems, resources are typically highly constrained, making tradeoffs with computational precision a fundamental concern. Indeed with increasing requirements on algorithmic performance, many systems are trending toward higher computational precision to ensure accuracy of results. A low pass floating point FIR filter was used for de-noising and smoothing the sampled data. FIR filter is a digital filter, it operates on discrete time signals which have several advantages over analog filters. They are more accurate, highly immune to noise, easy to implement and inexpensive to change the operating characteristics like cutoff frequency. These characteristics are important in electro-chemical applications where most signals have low frequencies that might get distorted due to the drift in the analog circuit. FIR filter output is computed non-recursively as a weighted, finite term sum of past and present values of the input as following equation.

The CPU save average value of 1024 filtered samples in the flash memory. The subtraction of each two averaged value of pulses make one point of voltammogram. Furthermore, to optimize performance and accomplish low power consumption, during sampling and conversion of signals by the ADC, DMA (Direct Memory Access) has been utilized to disable the CPU:

$$
\mathrm{w}(\mathrm{n})=0.54-0.46 \operatorname{Cos}(2 \pi \mathrm{n} /(\mathrm{N}-1))
$$

To suppress the above $40 \mathrm{~Hz}$ interference in ASDPV signal, a low-pass filter is designed whose coefficients are obtained from online tool (TFilter). The normalized frequency response of the designed low-pass FIR filter is shown in Fig. 4. This filter is designed using the Hamming window which is a fixed window, thus, only one independent parameter, window length is required which controls the main-lobe width. Also, the Hamming window is comparatively stable and has less ripples in low-pass in comparison to Rectangular and Hanning window (Wang, 2004) and thus is the preferred window for analysis. The Hamming window function is given by Eq. 2:

$$
\mathrm{w}(\mathrm{n})=0.54-0.46 \operatorname{Cos}\left(\frac{2 \pi \mathrm{n}}{\mathrm{N}-1}\right)
$$

The ARM Cortex processor has a large variety of highly efficient signal processing features. NXP Semiconductors provide DSP libraries for ARM Cortex-M3 CPU. Cortex Microcontroller Software Interface Standard (CMSIS) is a vendor-independent hardware abstraction layer for the Cortex processors. The CMSIS DSP Library provides the embedded filter algorithms contains FIR filtering routines which is implemented on the device for ASDPV filtering (Fig. 5).

Multiple peaks detection and metal identification: Voltammogram peaks have useful topological features which the position of peak shown the standard potential (X axis) that is unique for identification of the metals and the height of peak ( $\mathrm{Y}$ axis) is proportional to their concentration. A common requirement in ASDPV data processing is to detect peaks in the voltammogram signal and measure their positions and heights. The technique 

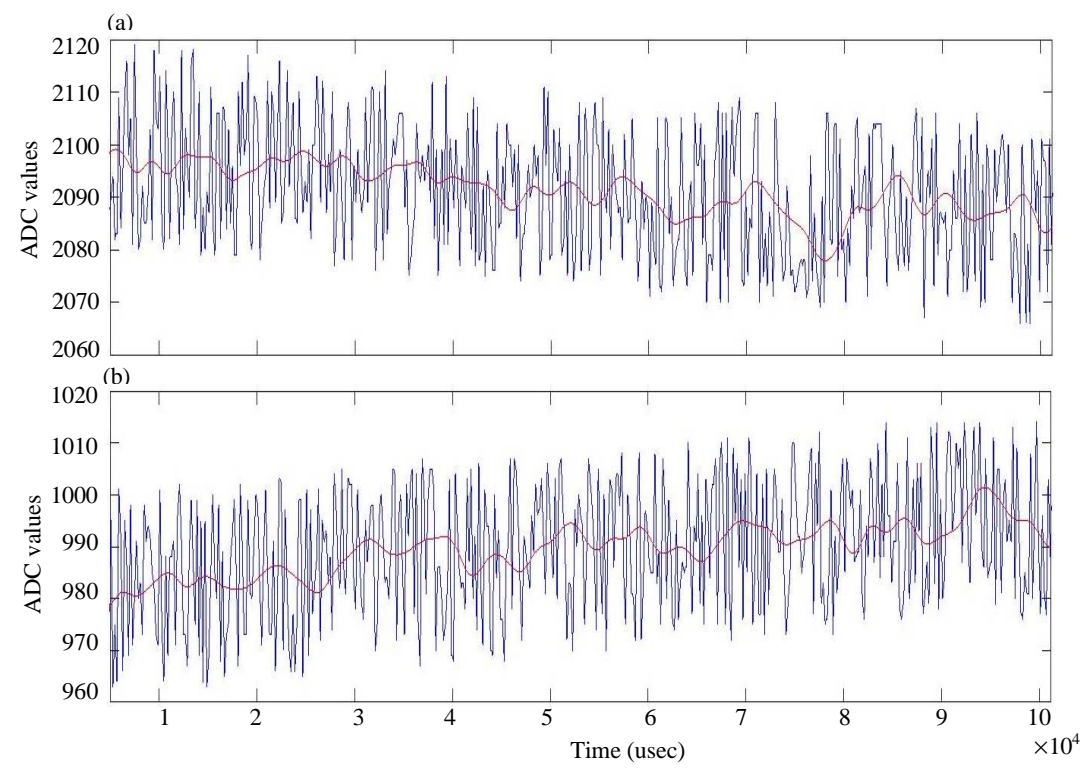

Fig. 5: The effect of FIR filter on the potentiostat samples during up and down pulses: a) Original sample 1 signal vs. low pass FIR filtered signal and b) Original sample 2 signal vs. low pass FIR filtered signal

described here is to recognize that a peak is the highest point between valleys. What makes a peak is the fact that there are lower points around it. This strategy is adopted to look for the highest point, around which there are points lower by $\mathrm{X}$ on both sides. So, a point is selected as a peak if:

$$
(y(t)-y(t-d t)>m) \text { and }(y(t)-y(t+d t)>m)
$$

where, $\mathrm{dt}$ and $\mathrm{m}$ are parameters for time-delay and sensitivity control, respectively. After peaks finding, device can determining the metal ions based on the position of the peaks. Because of existence of background current or other ions inside the solution, the voltammogram may have different peaks nearby or overlapped the desired peak of ion. In many applications, overlapped peaks resolution problem of detected signal becomes a bottleneck to restrict the development of automation for online in-situ analyzing device. The instrumental methods cannot absolutely resolve overlapping peaks; consequently, embedded signal processing needs to be implemented on the MCU. On the aspect of detection method for metal ions, Gaussian distribution is a very commonly occurring continuous Probability Distribution Function (PDF). Gaussian (Normal) distributions are extremely important in statistics and are often used in the chemistry for real-valued random variables whose distributions are not known.

A peak separation approach based on Gaussian distribution has been formulated by using the statistic values mean $\left(\mu_{\mathrm{i}}\right)$ and standard deviations $\left(\boldsymbol{\delta}_{\mathrm{i}}\right)$ of sampled oxidation voltage. For identification of an unknown metal the peak voltage of sampled voltammogram is compared versus a Probability Density Function (PDF) (Christidis et al., 2007; Harvey, 2000) by using the following equation:

$$
P\left(E_{p}\right)=\frac{1}{\delta i \sqrt{2 \delta i}} e^{\frac{(E \mathrm{p}-\mu \mathrm{i})^{2}}{2 \delta i^{2}}}
$$

A metal identifying by device if the voltage of detected peak (Epeak) have the highest probability when compared against the probability density function of all metals stored in a database. Additionally, the height of voltammogram peak is directly proportional to concentration of that component. So, the concentration predicting can be done by comparing the magnitude of peak with the stored calibration equations of the different metals in system memory.

\section{RESULTS AND DISCUSSION}

This study describes the technique for the electrochemical measurement and signal processing results of sampled data. In order to test the device functionality, heavy metal experiments have been done. To run the electrochemical experiments need prepare the solution. Solutions are often make ready by diluting a much more concentrated available solution with an electrolyte and de-ionized water. The potassium chloride (KCL) was used inside the solution as supporting electrolyte (electrolyte) have free electron to make electron exchange in the electrochemical cell easer. For 




Fig. 6: The result voltammogram of $0.5 \mathrm{ppm}$ of $\mathrm{Pb}$ with using FIR filter; DPV $\mathrm{Pb} 2+(0.5 \mathrm{ppm})$

$0.1 \mathrm{~m} \mathrm{KCl}$ supporting electrolyte preparation $0.7455 \mathrm{~g}$ mass of $\mathrm{KCl}$ is mixed with $100 \mathrm{~mL}$ de-ionized water. Cadmium, lead and zink as most dangerous heavy metals selected for tests with 1000 ppm commercial available concentration. Solid screen-printed electrodes utilized as sensor for chemical experiments.

The device testing was performed by applying differential pulse anodic stripping voltammetry signal on sensor inside the solutions with several concentrations. The device was tested with $0.5-10 \mathrm{ppm}$ solutions. The solutions were tested with the differential pulse was swiped between -1.5 and $+1.5 \mathrm{~V}$.

A burst sampling has been done at the end of each pulses. Sampled data has been sent to the FIR filter to remove noise and interference. In order to shown the effect of FIR filter on sampling data, the 1024 samples on each half cycle interval and filtered signals has been saved using debugging tools. Figure 6 shows the normal signal and filtered signal of ADC values in last $10 \mathrm{uS}$ of pulses that comparing together. The results shown that by converting the $\mathrm{ADC}$ binary sampled data to equivalent voltage, the results without FIR filtering are varying in range of $45 \mathrm{mV}$. This voltage range is about $1 \mathrm{uA}$ error of potentiostat current which is not acceptable and have bad effect on peak detection algorithm. The implemented FIR filter improves this range of error to have more clear and precise peaks at obtained voltammogram.

The effect of proposed sampling strategy and using FIR filter on the voltammetry of a $\mathrm{Pb}$ with $0.5 \mathrm{ppm}$ concentration is depicted at Fig. 7. It shown how using oversampling and FIR filter can improve the



Fig. 7: Histogram and PDF of peak potential for lead ion samples (Probability density function)

voltammogram shape and decrease the errors on finding the peak's locations. For statistical parameter calculation, thirty six independent measurements of the electrical potential and peak current amplitude of lead with different concentration were recorded. It gives the $\mu=-524$ and $\sigma=9.5$ values for mean and standard deviation of lead, respectively. In order to examine how the calculated potentials peaks follow the Gaussian density function, a chi square goodness of fit test has been done. Table 2 and 3 shown the results of this test with the null hypothesis that data have a Gaussian shape. The Chi-square goodness of fit test with different $\alpha$ values from $0.2-0.01$ shown p-factor is equal to 0.91125 and cannot reject the null hypothesis. Figure 7 depicts the probability density function and histogram of $\mathrm{Pb}$ voltage 
Table 2: The Chi-squared goodness of fit test results of peak potential for lead ion samples

\begin{tabular}{|c|c|c|c|c|c|}
\hline Cronbach's $\alpha$ & \multicolumn{3}{|c|}{ Critical values } & & Reject \\
\hline 0.2 & \multicolumn{3}{|c|}{4.6416} & & No. \\
\hline 0.1 & \multicolumn{3}{|c|}{6.2514} & & No. \\
\hline 0.05 & \multicolumn{3}{|c|}{7.8147} & & No. \\
\hline 0.02 & \multicolumn{3}{|c|}{9.8374} & & No. \\
\hline 0.01 & \multicolumn{3}{|c|}{11.3450} & & No. \\
\hline \multicolumn{6}{|c|}{ Chi-squared; Degree of freedom; 3 Statistic 0.53451 ; p-value 0.91125} \\
\hline Test preparations & $t_{\text {peak }}(h)$ & $\mathrm{C}_{\max }(\mathrm{ng} / \mathrm{mL})$ & $\mathrm{AUC}_{0-8}(\mathrm{ng} / \mathrm{mL} \cdot \mathrm{h})$ & $\mathrm{k}_{e}\left(\mathrm{~h}^{-1}\right)$ & $\mathrm{k}_{\mathrm{a}}\left(\mathrm{h}^{-1}\right)$ \\
\hline Nimotop $^{\mathrm{TM}}$ & 0.58 & 80.29 & 234.70 & 0.376 & 14.832 \\
\hline Immediate release & 0.59 & 70.02 & 277.33 & 0.302 & 5.058 \\
\hline Sustained release & 3.74 & 28.92 & 225.53 & 0.284 & 0.442 \\
\hline
\end{tabular}

peaks. From the results of the experiments it has been found that the lowest concentrations of heavy metals that can be identified by the device are 0.5 ppm. Figure 7 shows the differential pulse voltammograms of different metals at their 1 ppm concentration obtained using device. To verify the ability of the system's simultaneous identification algorithm, an experiment in which a solution containing all three metals was prepared. Figure 6 shows the DPV voltammogram attained with the $5 \mathrm{ppm}$ concentration of all three metals that a peak corresponding to each of them can be clearly seen satisfactory.

\section{CONCLUSION}

Miniaturization of analytical instruments is the primary goal of device development at these days. Determination of specific and group of compounds is the point of view of this improvement. Enhancing low detection limits and offering online and in-situ monitoring of environment are needed. In this study, we have presented the embedded algorithms, designed as a portable electrochemical transduction system with integrated recording and data manipulation. It potentially could replace computer systems in standard electrochemical setups. The device can deliver the smooth and noise free voltammogram of heavy metals as expected. The device can generate an excitation signal of ASDPV from $-3 \mathrm{~V}$ to $+3 \mathrm{~V}$ and produced a peaked voltammogram and analyze the solution by using embed online peak detection and Gaussian equation algorithm. The design of device and its ASDPV algorithm is verified.

\section{REFERENCES}

Bard, A.J. and L.R. Faulkner, 1980. Electrochemical Methods: Fundamentals and Applications. Wiley, Hoboken, New Jersey, USA., ISBN:9780471055426, Pages: 718.
Cheng, C.C., M.S. Young, S.W. Young and C.L. Chuang, 2002. An intelligent digital voltammetric system with multiple functions executed through stand-alone operation or PC-control. Biomed. Eng. Appl. Basis Commun., 14: 218-236.

Christidis, K., P. Robertson, K. Gow and P. Pollard, 2007. Voltammetric in situ measurements of heavy metals in soil using a portable electrochemical instrument. Meas., 40: 960-967.

Dorokhin, D., G.A. Crespo, M.G. Afshar and E. Bakker, 2014. A low-cost thin layer coulometric microfluidic device based on an ion-selective membrane for calcium determination. Anal., 139: 48-51.

Fischer, L.M., M. Tenje and A. Boisen, 2011. Development of an electrochemical-cantilever hybrid platform. Ph.D Thesis, Technical University of Denmark (DTU), Kongens Lyngby, Kingdom of Denmark.

Francis, M.F., L. Lavoie, F.M. Winnik and J.C. Leroux, 2003. Solubilization of cyclosporin a in dextran-g-polyethyleneglycolalkyl ether polymeric micelles. Eur. J. Pharm. Biopharm., 56: 337-346.

Ghoreishizadeh, S.S., I. Taurino, S. Carrara and G. De Micheli, 2012. A current-mode potentiostat for multi-target detection tested with different lactate biosensors. Proceedings of the 2012 IEEE International Conference on Biomedical Circuits and Systems (BioCAS), November 28-30, 2012, IEEE, Hsinchu, Taiwan, ISBN:978-1-4673-2291-1, pp: 128-131.

Harvey, D., 2000. Electrochemical Methods. In: Modern Analytical Chemistry, Harvey, D. (Ed.). McGraw-Hill, New York City, USA., ISBN:9780072375473, pp: $461-542$.

Honeychurch, K.C., 2012. Screen-printed electrochemical sensors and biosensors for monitoring metal pollutants. Insci. J., 2: 1-51. 
Kazi, T.G., H.I. Afridi, F. Shah, S.S. Arain and K.D. Brahman et al., 2016. Simultaneous determination of silver and other heavy metals in aquatic environment receiving wastewater from industrial area, applying an enrichment method. Arabian J. Chem., 9: 105-113.

Singh, A., A. Goyal, T.K. Saravanan and G.B. Iyer, 2008. An electrochemical amperometric sensing circuit with wireless interface for detection of neural signals. ECE. Gatech. Edu., 1: 13-15.
Strasunske, K., 2010. Automatic monitoring systems for trace metals in natural and waste water. Ph.D Thesis, Norwegian University of Science and Technology, Trondheim, Norway.

Wang, J., 2004. Analytical Electrochemistry. 2nd Edn., Wiley, Hoboken, New Jersey, USA., ISBN: 9780471 460794, Pages: 232.

Zoski, C.G., 2007. Handbook of Electrochemistry. Elsevier, Amsterdam, Netherlands, ISBN:9780444519580, Pages: 892. 